\title{
Ruptura de vena cava inferior secundaria a uso de sonda de ecografía intracardiaca tratada de manera exitosa con balón de alta distensibilidad
}

\author{
Juan Carlos Díaz Martínez, María del Mar Serna Posada, William Uribe Arango, \\ Jorge Eduardo Marín Velásquez y Mauricio Duque Ramírez*
}

\author{
Cardiología y Electrofisiología, Universidad CES, Medellín, Colombia
}

Recibido el 18 de junio de 2018; aceptado el 22 de abril de 2019

Disponible en Internet el 8 de julio de 2019

\section{PALABRAS CLAVE}

Ablación/aislamiento de venas pulmonares; Lesión;

Perforación;

Ruptura;

Vena cava inferior

\section{KEYWORDS}

Pulmonary vein ablation / isolation;

Lesion;

Perforation;

Rupture;

Inferior vena cava

\begin{abstract}
Resumen La ruptura de la vena cava inferior durante los procedimientos de intervencionismo percutáneo es una complicación infrecuente que se asocia con alta tasa de mortalidad aunque sea detectada a tiempo y se realice reparo quirúrgico, el cual es hoy el estándar de manejo. No existen hasta el momento casos reportados de manejo percutáneo de perforación de la vena cava durante procedimientos de electrofisiología. Se describe el caso de una paciente llevada a aislamiento eléctrico de venas pulmonares para el manejo de fibrilación auricular paroxística, en quien, durante el procedimiento, se produjo perforación accidental de la vena cava inferior con la sonda de ecocardiografía intracardiaca, la cual fue tratada exitosamente mediante el uso de un balón de alta distensibilidad con lo que se logró adecuada hemostasia sin necesidad de intervención quirúrgica. Se considera que el uso de un balón de alta distensibilidad puede ser una herramienta útil en el control del sangrado asociado a lesiones vasculares iatrogénicas, y que por consiguiente todo intervencionista debería tener presente.

(c) 2019 Sociedad Colombiana de Cardiología y Cirugía Cardiovascular. Publicado por Elsevier España, S.L.U. Este es un artículo Open Access bajo la licencia CC BY-NC-ND (http:// creativecommons.org/licenses/by-nc-nd/4.0/).
\end{abstract}

Inferior vena cava rupture associated with the use of an intracardiac echocardiography probe and successfully treated with a high-compliance balloon

Abstract Rupture of the inferior vena cava during percutaneous intervention procedures is an uncommon complication. It is associated with a high rate of mortality, even when it is detected at the time and the current standard management, surgical repair is performed. At present there are no cases reported of the percutaneous management of a vena cava perforation during electrophysiology procedures. The case is described of a patient subjected to electric ablation of pulmonary veins for the management of paroxysmal atrial fibrillation. During the

\footnotetext{
* Autor para correspondencia.

Correo electrónico: mauricioduquemd@gmail.com (M. Duque Ramírez).
} 
procedure there was an accidental rupture of the inferior vena cava with the echocardiography cardiac catheter. She was successfully treated using a high-compliance balloon, with adequate haemostasis being achieved without surgical intervention. The use of a high-compliance balloon is considered as a useful tool in the control of bleeding associated with iatrogenic vascular injuries, and for this reason all interventionist should be aware of it.

(c) 2019 Sociedad Colombiana de Cardiología y Cirugía Cardiovascular. Published by Elsevier España, S.L.U. This is an open access article under the CC BY-NC-ND license (http:// creativecommons.org/licenses/by-nc-nd/4.0/).

\section{Introducción}

En la década de los 60 , las lesiones que comprometían la vena cava se asociaban con una mortalidad del 41 al $80 \%$, cifra que descendió hasta el $30 \%$ en la década de los setenta en centros especializados en trauma, gracias a un transporte adecuado, atención prehospitalaria de calidad y resucitación oportuna. Aún en la actualidad, las lesiones de la vena cava tienen un pronóstico ominoso, en razón a su alta morbilidad y mortalidad ${ }^{1,2}$.

La mayor parte de las lesiones de la cava descritas en la literatura se asocian a trauma (principalmente penetrante). Dichas rupturas se clasifican de acuerdo con su localización anatómica en suprahepáticas, retrohepáticas, perirrenales e infrarrenales. Mientras que la lesión de vena cava inferior en su porción infrarrenal es la más común, las de las porciones retrohepática y suprahepática son las más letales dada la dificultad que conlleva el hecho de exponer y evaluar el sitio donde ocurren ${ }^{3}$. No obstante, la incidencia y las cifras de mortalidad de la ruptura iatrogénica de la vena cava inferior durante procedimientos de intervencionismo percutáneo (incluyendo procedimientos de electrofisiología), no han sido descritas en la literatura médica. De igual forma, no existen hasta el momento casos reportados de manejo endovascular de una perforación de la vena cava inferior durante un procedimiento de electrofisiología.

\section{Caso}

Mujer de 69 años, con antecedente de hipertensión arterial y fibrilación auricular paroxística, llevada a aislamiento eléctrico de venas pulmonares bajo anestesia general, en quien se realizó abordaje femoral bilateral, con dos introductores $5 \mathrm{~F}$ en el lado derecho (los cuales serían intercambiados posteriormente por introductores transeptales) y en el lado izquierdo con un introductor $11 \mathrm{~F}$ de $30 \mathrm{~cm}$ (Fastcath $^{T M}$, St Jude Medical, Saint Paul, Minnesota, Estados Unidos). Una vez realizadas las punciones, se procedió a infundir 5.000 unidades de heparina no fraccionada intravenosa. A través del introductor $11 \mathrm{~F}$ femoral izquierdo se avanzó una sonda de ecocardiografía intracardiaca (ecocardiografía intracardiaca) Viewflex Xtra ${ }^{T M}$ (St Jude Medical, Saint Paul, Minnesota, Estados Unidos) bajo visualización fluoroscópica, protruyendo por fuera del introductor $11 \mathrm{~F}$ sin encontrar resistencia significativa durante el avance. No obstante, no fue posible avanzar más allá de la silueta hepática a pesar de maniobrar la sonda, motivo por el cual se decidió realizar una venografía a través del mismo introductor 11F. Se evidenció de esta manera una perforación de la vena cava inferior, con salida de la sonda de ecocardiografía intracardiaca hacia el espacio retroperitoneal (fig. 1). Una vez documentado esto, se revirtió el efecto de la heparina con protamina, se retiró la sonda de ecocardiografía intracardiaca y se ubicó el sitio exacto de la perforación, observándose salida del medio de contraste hacia el espacio retroperitoneal. Se decidió, entonces, avanzar una guía de alto soporte Amplatz Super Stiff ${ }^{T M}$ (Boston Scientific, Marlborough, Massachussets, Estados Unidos) 0.035' a través de uno de los introductores femorales derechos, el cual se intercambió por un introductor 10F, para posteriormente avanzar un balón de alta distensibilidad (Reliant ${ }^{\top M}$, Medtronic, Minneapolis, Minnesota, Estados Unidos) hasta el sitio de la perforación. Una vez ubicado, se infló de manera progresiva hasta lograr la oclusión del defecto de la pared (fig. 2). Se realizaron varios inflados de 5-10 minutos cada uno, con 1 minuto de espera entre cada inflado. Con los inflados se logró una disminución progresiva de la cantidad de medio de contraste que fluía por la perforación, hasta lograr hemostasia sin necesidad de intervención quirúrgica (fig. 3). Al día siguiente de la intervención se realizó una angiotomografía de abdomen, en la cual no se observaron alteraciones anatómicas que explicaran la perforación. La paciente fue dada de alta 3 días después con terapia anticoagulante (apixabán $5 \mathrm{mg}$ vía oral cada 12 horas) y continúa en buenas condiciones luego de dos años.

\section{Discusión}

En la literatura existen reportes de ruptura iatrogénica de la vena cava inferior, en algunos de los cuales el uso de balones ha sido efectivo para el control de sangrado ${ }^{1,4,5}$. No obstante, previamente no se ha descrito la oclusión con balón en el caso de perforación asociada a procedimientos intervencionistas, particularmente de electrofisiología. En la mayoría de los casos reportados se realizó manejo quirúrgico, lo cual se asocia con morbilidad y mortalidad significativas, mientras que en algunos casos en los cuales se dio manejo endovascular se usaron stents, lo que a su vez trae consigo riesgos adicionales, como migración del dispositivo y restenosis/trombosis lo que implicaría antiagregación y/o anticoagulación subsecuente, en el contexto de paciente con lesión vascular. 


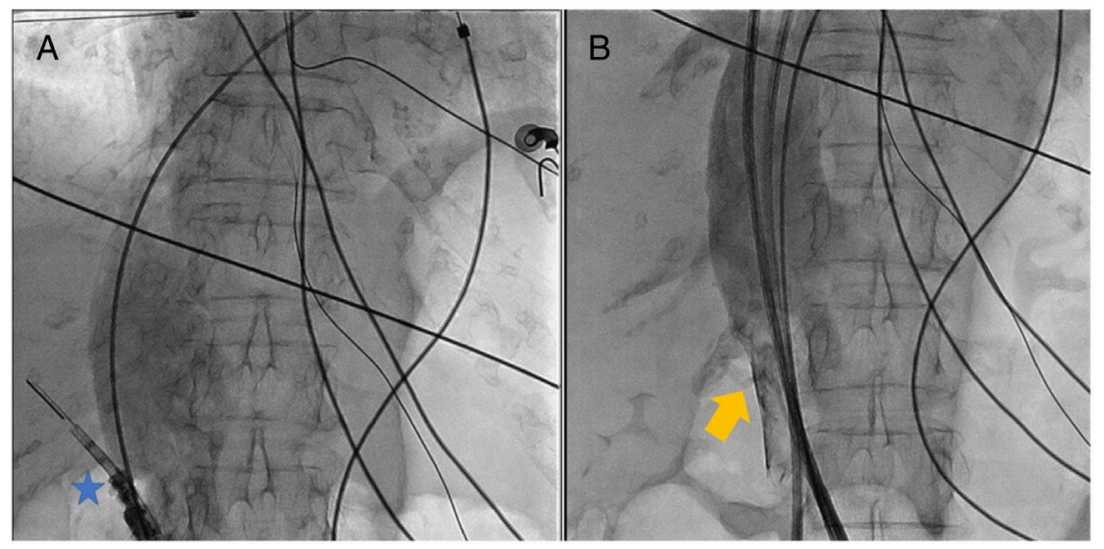

Figura 1 Imagen inicial de la perforación de la vena cava. A. Protrusión del catéter de ecocardiografía intracardiaca a través de la pared de la vena cava inferior (estrella azul). Se observa medio de contraste extravasado en el trayecto del catéter. B. Inyección de contraste una vez retirado el catéter; se observa salida del medio de contraste hacia el espacio retroperitoneal (flecha amarilla).

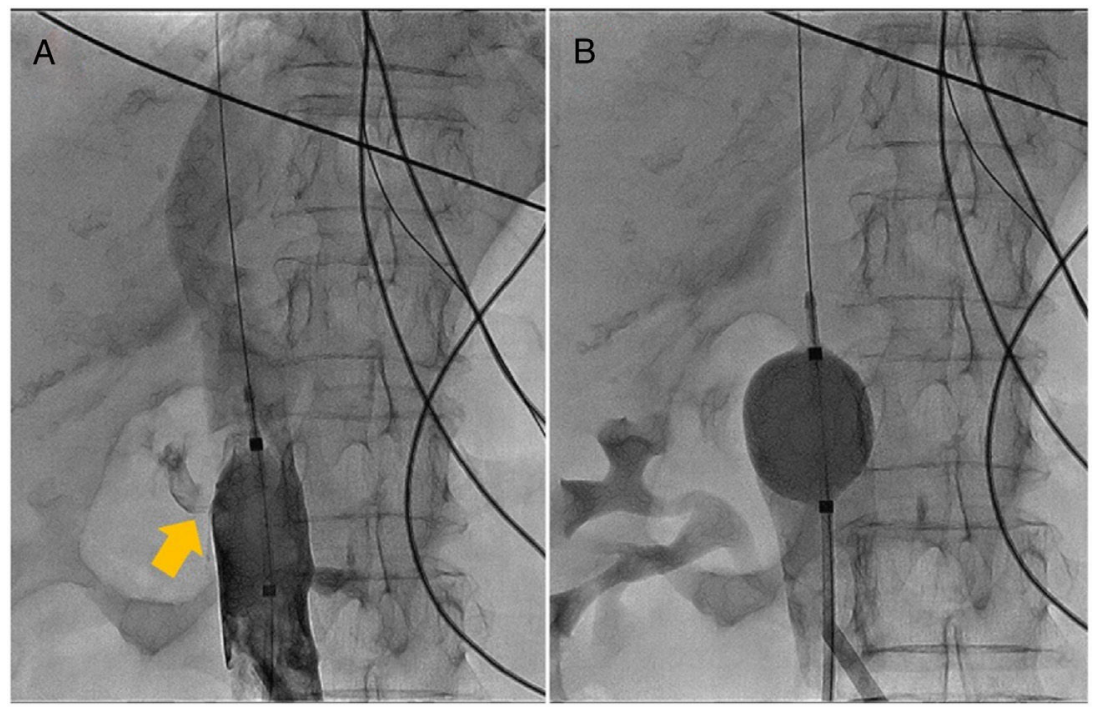

Figura 2 Proceso de inflado del balón hasta lograr la oclusión del defecto. A. Con el inflado parcial inicial se logra ubicar de manera precisa el sitio de la perforación. Este inflado parcial no ocluye el orificio y aún permite la salida de medio de contraste (flecha amarilla). B. Al inflar más el balón se logra la hemostasia (no se observa salida del medio de contraste). Cada uno de estos inflados se realizó por periodos de 5 a 10 minutos, con un periodo de un minuto entre inflado.

Pese a que la ruptura iatrogénica de la vena cava inferior es poco común, queda claro que es un escenario potencialmente fatal. Estudios animales han demostrado disminución en la pérdida sanguínea y en la mortalidad asociada al uso de balón de alta distensibilidad para el manejo de lesiones de la vena cava inferior, así como mejoría en los parámetros de hipoperfusión (hipotermia, lactato, acidosis) ${ }^{6}$. Teniendo en cuenta que el número y la complejidad de las intervenciones percutáneas viene en aumento y que muchas de estas involucran el uso de catéteres e introductores rígidos y de gran calibre, es posible que la cantidad de pacientes que presenten perforación de la vena cava inferior durante procedimientos de intervencionismo percutáneo (incluyendo procedimientos de Electrofisiología,
Hemodinamia o Radiología Intervencionista) aumente, por lo cual se cree que este caso brinda a los médicos la posibilidad de ofrecer al paciente una intervención que potencialmente podría salvarle la vida.

Específicamente en Electrofisiología, se sabe que las laceraciones de la vena cava superior son una de las complicaciones más letales durante la extracción de electrodos de dispositivos cardíacos implantables, con una tasa de mortalidad de hasta el $50 \%$, secundaria a hemorragia masiva ${ }^{7,8}$. Recientemente, se lanzó al mercado un balón de alta distensibilidad diseñado solo para ser usado en casos de ruptura de la vena cava superior durante procedimientos de extracción de electrodos. Al ocluir temporalmente el vaso comprometido, se logra disminuir la pérdida sanguínea y ganar tiempo 


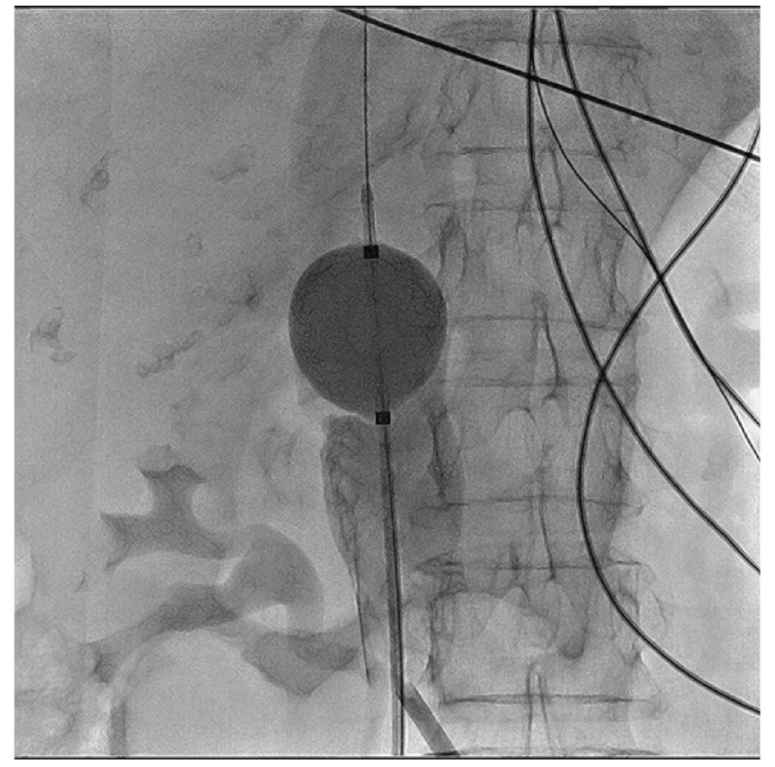

Figura 3 Una vez lograda la hemostasia se desplazó el balón y se infló nuevamente por encima del sitio de la perforación, con el fin de hacer una nueva inyección de contraste con la vena cava ocluída casi en su totalidad en búsqueda de fugas remanentes. Al no observar fugas, se consideró que la hemostasia había sido adecuada.

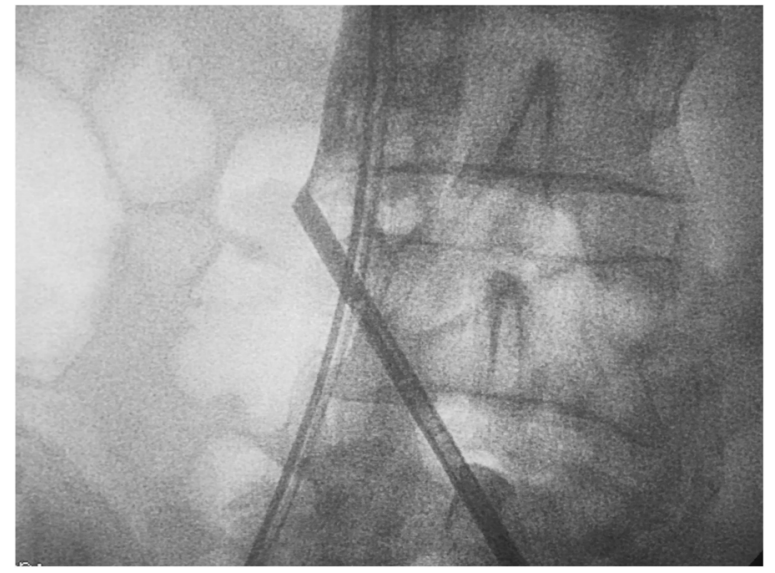

Figura 4 Imagen que corresponde a otro paciente. Se observa un introductor $11 \mathrm{~F}$ ingresando a la cava desde la vena ilíaca izquierda. Por la longitud del introductor en relación con el tamaño del paciente, el introductor queda posicionado justamente sobre la pared lateral de la vena cava, empujándola hacia afuera ("tenting"). Avanzar el catéter de ecocardiografía intracardiaca con el introductor en esta posición, podría llevar a perforación inadvertida de la vena cava inferior.

antes del colapso hemodinámico del paciente, permitiendo su traslado a cirugía en mejores condiciones, lo que a su vez se asocia con menor mortalidad ${ }^{8}$.

Respecto al motivo de la ruptura, los autores creen que estuvo relacionada con el uso de un introductor de $30 \mathrm{~cm}$. Aunque este facilita el avance de la sonda desde la ingle izquierda hasta la vena cava (ya que en algunos casos la tortuosidad de las venas femorales e ilíacas puede dificultar el avance de la sonda), en evaluaciones posteriores a este caso se halló que en algunos pacientes su longitud permite que la punta empuje sobre la pared de la cava, haciendo una especie de "tenting". Esto a su vez facilita que con una mínima presión ejercida por el catéter de ecocardiografía intracardiaca (el cual es rígido por naturaleza y de punta relativamente delgada) haya perforación de la vena (fig. 4). De manera alterna, es posible que el introductor haya sido el que perforó la vena cava inferior al quedar su punta desprotegida (es decir, una vez retirada la guía y el dilatador utilizados para avanzarlo) contra la pared de la vena cava inferior. Esto también explicaría la falta de resistencia percibida durante el avance de la sonda de ecocardiografía. Ante este hallazgo, los autores modificaron la manera en la cual se avanza la sonda de ecocardiografía intracardiaca por fuera del introductor; en la actualidad, la avanzan hasta llegar a unos dos centímetros de la punta del introductor, para posteriormente retirarlo, descamisando la sonda sin avanzarla. De esta manera, la punta queda libre en el espacio intravascular, permitiendo su manipulación con el fin de alinearla de manera coaxial con el eje mayor de la vena, lo que quizá evitaría a futuro nuevas perforaciones inadvertidas.

\section{Conclusión}

La ruptura de vena cava inferior es un evento catastrófico para el paciente, dada su alta morbilidad y mortalidad asociadas. Aunque en la literatura existen casos notificados en los cuales se acude al uso de terapia percutánea, en todos ellos posteriormente proceden a corrección quirúrgica abierta o implante de stent, de modo que la aproximación descrita aquí (hemostasia mediante el uso de compresión con balón), es novedosa. Aunque la corrección quirúrgica del defecto por lo general es el estándar de manejo, el uso de balones de alta distensibilidad es una alternativa para el médico intervencionista.

\section{Financiación}

Ninguna.

\section{Conflicto de intereses}

Ninguno.

\section{Bibliografía}

1. Briggs CS, Morcos OC, Moriera CC, Gupta N. Endovascular treatment of iatrogenic injury to the retrohepatic inferior vena cava. Ann Vasc Surg. 2014;28:1794, e13-5.

2. Ravikumar S, Stahl WM. Intraluminal balloon catheter occlusion for major vena cava injuries. The Journal of trauma. $1985 ; 25: 458-60$.

3. Bui TD, Mills JL. Control of inferior vena cava injury using percutaneous balloon catheter occlusion. Vascular and Endovascular Surgery. 2009;43:490-3.

4. Castelli P, Caronno R, Piffaretti G, Tozzi M. Emergency endovascular repair for traumatic injury of the inferior vena cava. Eur $\mathrm{J}$ Cardiothorac Surg. 2005;28:906-8.

5. Willaert W. Van HerzeAnn Vasc Sug. 2012;26:733, e1-4

6. Reynolds CL, Celio AC, Bridges LC, Mosquera C, O'Connell B, Bard $M R$, et al. REBOA for the IVC? Resuscitative balloon occlusion of the inferior vena cava (REBOVC) to abate massive hemorrhage 
in retrohepatic vena cava injuries. The Journal of Trauma and Acute Care Surgery. 2017;83:1041-6.

7. Espinel CF, Calligaro KD, Dougherty MJ. Percutaneous balloon occlusion of the inferior vena cava as an adjunct for treating ruptured type IV thoracoabdominal aneurysm and aortocaval fistula. J Vasc Surg. 2006;43:834-5.
8. Azarrafiy R, Tsang DC, Boyle TA, Wilkoff BL, Carrillo RG. Compliant endovascular balloon reduces the lethality of superior vena cava tears during transvenous lead extractions. Heart Rhythm. 2017;14:1400-4. 